\title{
Multilevel computer-generated holograms for reconstructing 3-D images in combined optical-digital security devices
}

\author{
V.I. Girnyk ${ }^{1)}$, S.O. Kostyukevych ${ }^{2)}$, P.Ye. Shepeliavyi ${ }^{2)}$, A.V. Kononov ${ }^{1)}$, I.S. Borisov ${ }^{1)}$ \\ 1) STC "Optronics", LTD, Kiev Taras Shevchenko University, Radiophysical Dept., 2 build 5 prospekt acad. Glushkov, 03137 Kyiv, Ukraine; \\ Phone: +380 (44) 266 9235; phonelfax:+380 (44)252 5597; e-mail: optronics@ukr.net \\ 2) Institute of Semiconductor Physics, NAS of Ukraine, 45 prospekt Nauky, 03028 Kyiv, Ukraine
}

\begin{abstract}
Computer-generated holograms (CGHs) integrated within combined optical-digital security devices (CO/DSDs) are described in this work. They can restore the monochrome and color 3D images in white light. To record them, the Electron Beam Lithography (EBL) is used. Our investigations on optimization of synthesis and recording the CO/DSDs with the integrated in it multilevel CGHs of 3D images possessed horizontal parallax only (HPO) are presented here. The CGH fabrication process is mainly composed of two parts: calculation of the interferogram data (ID) and their recording. Calculation of the ID is done as follows: firstly, the geometrical and optical constants of recording scheme and the object surface represented by the elemental self-radiating areas, are determined, secondly, the basic parameters accounting for discretization of ID in hologram plane is defined. The ID values can be derived by calculation of the necessary elemental object areas bipolar intensities sum. Next, over suitable quantization of ID, recording the rectangle data appropriate for EBL onto glass coated with non-organic photoresist based on $\mathrm{As}_{40} \mathrm{~S}_{40} \mathrm{Se}_{20}$ is performed. We have also investigated reciprocal influence of an optical part of the CO/DSD and a digital one.
\end{abstract}

Keywords: computer-generated holograms, Electron Beam Lithography, combined optical-digital security devices, interferogram data, 3D images, horizontal parallax only, multi color, bipolar intensity

Paper received 10.12.01; revised manuscript received 23.01.02; accepted for publication 05.03.02.

\section{Introduction}

The usage of holographic security devices (HSDs) for state and commercial needs considerably increases in recent decade. At the same, time the intensive development of holographic technologies and high level of activity in this area cause high probability of counterfeiting. In conditions mentioned, the elaboration of new methods of synthesis and recording information providing higher reliability against counterfeiting becomes more and more actual. The combined technology is one of the powerful modern protective methods of the HSDs synthesis. We had reported about creation of combined optical-digital security devices (CO/DSDs) utilizing both the optical and e-beam technologies at the SPIE/IS\&T conference "Electronic Imaging '2000" [1]. Based on the results of these investigations commerce production of HSDs of Combigrams ${ }^{\mathrm{TM}}$ type was started. Combigrams ${ }^{\mathrm{TM}}$ were oriented on protection of the most confidential documents and goods. They comprises electron-beam holography, optical holography and computer-generated hologram of $2 \mathrm{D}$ images. It allows them to differ from wide-spreaded and available dot matrix holograms and considerably reduce range of potential manufactures of the similar complexity HSDs. The usage of EBL technology with submicron resolution and of powerful computing means enable performing of the synthesis over Fresnel conditions and subsequent recording of computer-generated holograms that are able to reconstruct 3D images in white light. As against from CGH of 2D images calculated over Fourier conditions, they can be reconstructed in non-coherent light and can be identified on the first level of verification. It makes them attractive for further modification of Combigrams ${ }^{\mathrm{TM}}$. It is necessary to note that the synthesis and recording the Fresnel CGHs requires advanced technical means and considerable efforts, but in the case of successful embodiment of corresponding efforts it allows to hope for the commercial success. 


\section{V.I. Girnyk et al.: Multilevel computer-generated holograms ...}

\section{Essential principles of holographic synthesis}

\section{1. $3 D$ imaging on $2 D$ medium}

Correct reconstruction of color 3D image is possible only when a hologram is recorded on $3 \mathrm{D}$ registering medium, because of definite limitations characteristic for 2D registering medium. Therefore, in the case of the hologram recorded on the flat registering medium, to make it possible for the hologram to be reconstructed in white light according with rainbow holography principles, the angle of view in a definite direction must be considerably limited. It means that we lose some parallax, as a rule the vertical one, because of sight physiology stipulates necessity of keeping the horizontal parallax. So this work is devoted to synthesis of the Fresnel holograms that have only horizontal parallax only.

\subsection{Choice of synthesis methodology}

Ignoring the horizontal vertical parallax is equal to dividing the object space and holograms plane onto independent horizontal sections (Fig. 1). Within each similar section, points of the object that face into this section form an elementary hologram at the correspondent part of the hologram plane. When reconstructed, every elementary hologram (i.e., each independent section of the hologram plane) forms an image of the respective horizontal section for the object space. Calculations of an interference pattern for interaction of an object wave with the reference one only within it. Let us proceed to methods of such calculations.

First of all, we shall consider advantages of the hologram digital synthesis as compared to optical recording. Consider interaction of $\overline{A_{i}}$ wave radiated by some point object with plane reference wave $\bar{R}$ :

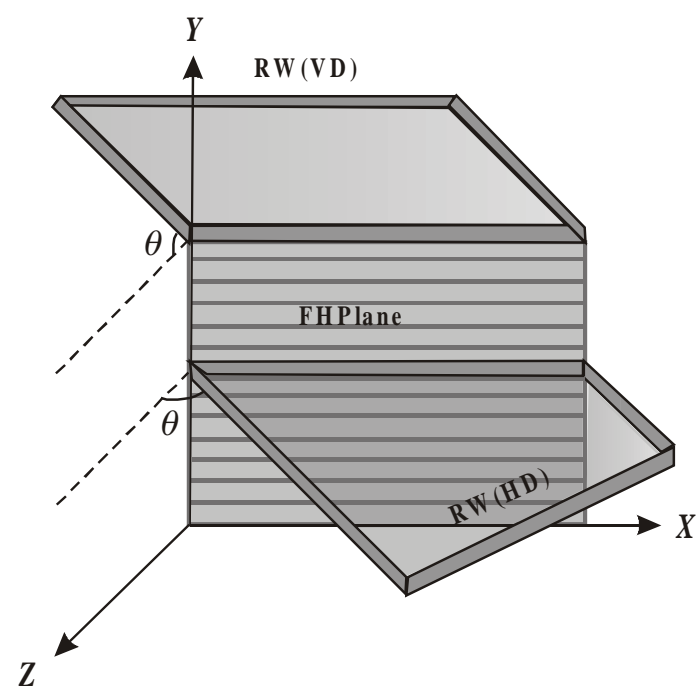

Fig. 1. Recording scheme geometry.
$\bar{A}_{i}=\frac{A_{i}}{\left|\vec{r}-\vec{r}_{i}\right|} \exp \left\{j\left[\vec{k}\left(\vec{r}-\vec{r}_{i}\right)+\varphi_{i}-\omega t\right]\right\}$.

$\bar{R}=R \cdot \exp \{-j k y \sin (\theta)-\omega t\}$,

- for the vertical direction of incidence (when $y=0$, the phase of reference wave is assumed to be zero);

$\bar{R}=R \cdot \exp \{-j k x \sin (\theta)-\omega t\}$,

-for the horizontal one (when $x=0$, the phase is equal to zero, too).

In optical recording, there is a possibility to register spatial distribution only for one characteristic, namely, for total intensity as a result of interference of all interacting waves:

$$
\begin{aligned}
& I(x, y)=\left|\bar{A}_{i}+\bar{R}\right|^{2}=\left(\bar{A}_{i}+\bar{R}\right)\left(\bar{A}_{i}^{*}+\bar{R}^{*}\right)= \\
& =\bar{R} \bar{R}^{*}+\bar{A}_{i} \bar{A}_{i}^{*}+\bar{A}_{i}^{*} \bar{R}+\bar{A}_{i} \bar{R}^{*} .
\end{aligned}
$$

Digital recording is effectively flexible and enables to register any term from the right part of the Eq. (4), or any superposition of them. The first term of the right part in Eq. (4) is simply some constant shift that reconstructs plane wave during restoring process. The second one is an autocorrelation term that describes autointerference. The third term is responsible for reconstruction of conjugated image using a wave that is conjugated with the reference one. And at last, the latter term describes reconstruction of the original image due to reference wave action. It would be reasonable to write it, but its complex character creates some obstacles. To record complex values, developed are several methods, but they need using printing devices with much higher resolution as compared to amplitude recording. By reason of a resolution limit, the most optimum seems to be registration of non-negative real value, especially in the case, when the printing device provides sufficient amount of quantization levels. There are several methods to choose this value. We shall use the most spread one, namely, we shall register, as suggested in [5], the value

polI $=K+b I$,

where

$b I=\bar{A}_{i}^{*} \bar{R}+\bar{A}_{i} \bar{R}^{*}=2 \operatorname{Re}\left\{\bar{A}_{i} \bar{R}^{*}\right\}$

is a bipolar intensity.

It follows that we shall calculated the next matrix

$b I\left(y_{m}, x_{n}\right)=b I(m, n)$,

where $m=0,1, \ldots, M-1$ is a number of a horizontal section, and $n=0,1, \ldots, N-1$ is a number of a vertical band within this section. For each section, we shall find minimum, maximum, means as well as root-mean-square values $b I$ for further adequate estimation of an optimum magnitude for quantization parameters including the constant shift $K$. 


\section{V.I. Girnyk et al.: Multilevel computer-generated holograms ...}

\subsection{Method for calculations of ID}

As mentioned above, the hologram together with an object space is divided onto independent sections. Consequently, the calculation of an interference pattern can be carried out step-by-step for each section.

First, contrary to [2], where an object was represented as a totality of light point sources, in this work, the object is defined as a totality of elementary radiating areas. This representation corresponds better to natural relations of observing real objects and should improve visual reception of restored images. Nevertheless, the developed program algorithm enables to calculate the interference pattern for point representation, too.

Second, as in [2], to reduce non-uniformities in the restored image, which are caused by essential differences of intensity ranges for various sections, the program allows to limit the angle of divergency for the object wave in the horizontal direction. This limitation is also useful due to decreasing the upper limit of a spatial frequency range, so frequencies of the interference pattern down to values that can be exactly reproduced by a printing device.

Let us determine limits of the spatial frequencies inherent to the hologram. They depend on a direction and angle of incidence $\theta$ for the reference wave as well as on a maximum angle of divergency $\alpha_{H P}$ for the object wave, that is a horizontal parallax angle (since the angle $\alpha$, at which the object wave spreads, is fixed in the range $\left.-\alpha_{H P}<\alpha<\alpha_{H P}\right)$.

For the horizontal incidence direction of the reference wave, the minimum spatial period in an inteferogram (which corresponds to the maximum spatial frequency) can be estimated using the formula:

$$
d_{\min }=\frac{\lambda}{\sin \theta+\sin \alpha_{H P}}
$$

When $\lambda=0.6 \mu \mathrm{m}, \theta=30^{\circ}, \alpha_{H P}=30^{\circ}$, we obtain $d_{\min }=0.600 \mu \mathrm{m}$, and assuming $\theta=45^{\circ}, \alpha_{H P}=45^{\circ}$ $d_{\min }=0.424 \mu \mathrm{m}$ for the same 1 value.

In the case of vertical incidence of the reference wave, the spatial period of the interference pattern can be defined as:

- in a vertical direction:

$$
d^{V}=\frac{\lambda}{\sin \theta}
$$

- as the minimum one in the horizontal direction:

$$
d_{\min }^{H}=\frac{\lambda}{\sin \alpha_{H P}}
$$

For the same values of parameters $(\lambda=0.6 \mu \mathrm{m}$, $\left.\theta=45^{\circ}, \alpha_{H P}=45^{\circ}\right)$ the formulae (9) and (10) yield in the next values of spatial periods: $d^{V}=d_{\min }^{H}=0.848 \mu \mathrm{m}$.
In accordance with the Kotelnicov-Shannon theorem about coding the analog signal, the spatial frequency of coding and recording an image should be at least twice as much the maximum spatial frequency presented in the image. It means that elementary horizontal and vertical intervals $D X$ and $D D Y$ should be smaller than the half of minimum spatial periods. For the horizontal incidence, direction of the reference wave $D D Y$ is not limited, $D X$ should not exceed $0.3 \mu \mathrm{m}$ for $\lambda=0.6 \mu \mathrm{m}$, and should not exceed $0.2 \mu \mathrm{m}$, when $\lambda=0.6 \mu \mathrm{m}, \theta=45^{\circ}, \alpha_{H P}=45^{\circ}$. In the case of vertical incidence and quantization parameters $\lambda=0.6 \mu \mathrm{m}, \theta=45^{\circ}, \alpha_{H P}=45^{\circ}, D X$ and $D D Y$ should not exceed the value $0.4 \mu \mathrm{m}$. (Accuracy of positioning and stump sizes of the accessible EBL is about $0.1 \mu \mathrm{m})$. It is worth to note that for normal operation of a diffraction grating, the latter must content at least 10-15 fringes. This limits the minimum value of the thickness for the horizontal section $D Y$ in the case of vertical incidence of the reference wave. Therefore, we chose $D Y_{\min }=20 \mu \mathrm{m}$, in order to set 23-24 diffraction fringes within the section, when $\theta=45^{\circ}$, and $16-17$ fringes at $\theta=30^{\circ}$. As to the horizontal incidence of the reference wave, there are no limitations on the lower limit of the value $D Y=D D Y$ for such geometry. At the same time, the upper limitation on the horizontal section thickness $D Y$ is determined by the desired higher resolution of a reconstructed image. The matter is that the spatial resolution in the object space in chosen to be equal to $D Y$.

Third, to eliminate a vertical-fringe structure, we shall prescribe a random initial phase to every elemental radiating area, as it have been proposed in previous investigations [2]. This way of randomizing initial phases transforms the interference pattern into a non-regular structure. In optical interpretation, this procedure is similar to placing a diffuser between the object and hologram, which eliminate clearly seen streak noise and replace it by less seen speckle noise.

Taking into account all above considerations, for each $n$-th vertical band $(n=0,1, \ldots, N-1)$ within the fixed $m$-th section $(m=0,1, \ldots, M-1)$, when the reference beam falls in horizontal direction, we summed up all contributions to the bipolar intensity (4) from each elementary radiating area belonging to the section. The contribution of one such radiating area can be represented by the next expression:

$$
C t b I=v i s \cdot \sqrt{\frac{s q A \cdot(\vec{n} \cdot \vec{r})}{r^{3}}} \cdot \cos \left( \pm k \cdot r+\varphi+\eta \cdot x_{F H}\right)
$$

where (as seen in Fig. 2) $x_{F H}=n \cdot D X$ is a horizontal coordinate of this vertical band; $\eta=k \cdot \sin \theta$ is a spatial frequency for the reference wave phase distribution in the hologram plane; $\varphi$-is an initial phase of the wave emitted by the area; $k=\frac{2 \pi}{\lambda}$ is a wavenumber; $r=\sqrt{r_{x}^{2}+z^{2}}$ is a modulus of vector that is drawn from this radiating area to the fixed vertical band in the hologram plane; $r_{x}=x_{F H}-x$ is a projection on this vector on the holo- 


\section{V.I. Girnyk et al.: Multilevel computer-generated holograms ...}

gram plane; $(\vec{n} \cdot \vec{r})=n_{x} \cdot r_{x}-n_{z} \cdot z$ is a projection of this vector on the normal to a given radiating area; $\vec{n}=\left(n_{x}, n_{y}, n_{z}\right)$ is a unity normal to an elementary area surface; $x, z$ are coordinates of the area; $s q A$ is a square of the wave amplitude emitted by this area, it characterizes its brightness; vis is a coefficient taking into account visibility of the area from the position of the $n$-th vertical band (i.e., reflect the degree of overlapping it by other areas); positive $(+)$ sign before $k \cdot r$ is chosen for elementary radiating areas placed below the hologram plane $(z<0)$, and negative $(-)$ sign is used for those placed above $(z>0)$. It is worth to note that the term

$$
\frac{(\vec{n} \cdot \vec{r})}{r}=\frac{n r \cos \angle(\vec{n}, \vec{r})}{r}=\cos \angle(\vec{n}, \vec{r}),
$$

which normalizing brightness $s q A$, is responsible for the natural behavior of this radiating area; for majority of natural light sources, flux emitted at an arbitrary direction $\vec{r}$ can be represented by the expression

$S=S_{0} \cos \angle(\vec{n}, \vec{r})$

where $S_{0}$ is a light flux emitted along the normal $\vec{n}$ to the source surface.

In the case of the vertically incident reference wave, for each $n$-th vertical band ( $n=0,1 \ldots, N-1)$, belonging to the $m$-th fixed section ( $m=0,1 \ldots, M-1)$, instead of one value of bipolar intensity one should calculate a respective massif with amount of elements

$$
\text { Counts } \leq \frac{D Y}{D D Y}
$$

where $D Y$ is a thickness of a horizontal section, and $D D Y$ is an elemental vertical interval.

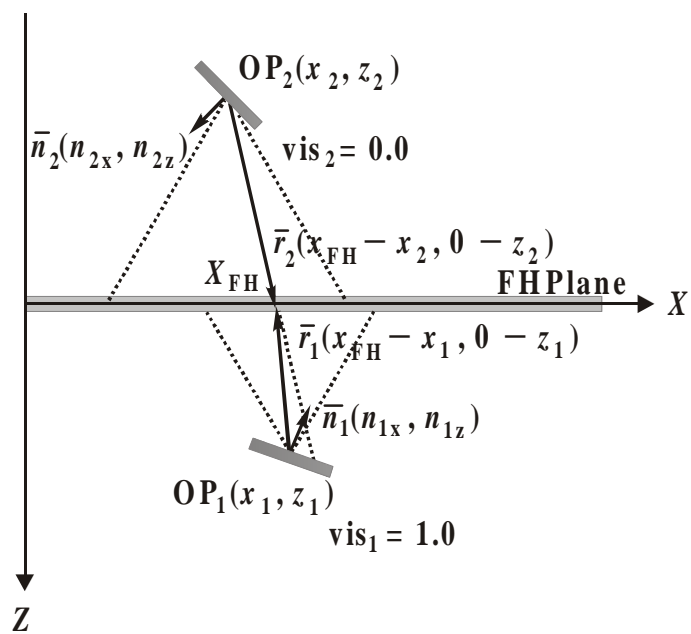

Fig. 2. Horizontal slice of object space and hologram plane: for calculation of definite bipolar intensity in the vertical band.
Thus, the elemental radiating area makes contribution as a massif with Counts elements, each of them being calculated using the formula

$$
\begin{aligned}
& C t b I_{i}=v i s \cdot \sqrt{\frac{s q A \cdot(\vec{n} \cdot \vec{r})}{r^{3}}} \cdot \cos \left( \pm k \cdot r+\varphi+\eta \cdot y_{i}\right), \\
& y_{i}=i \cdot D D Y, \quad i=0,1, \ldots, \text { Counts }-1
\end{aligned}
$$

The sign "+" before $\eta \cdot y_{i}$ corresponds to the reference wave incident from above.

Let us consider expressions (11) and (15) from (6) in detail. Eq. (6) foresees summing the next values in the hologram plane

$C t b I=2 \operatorname{Re}\left\{\bar{A} \bar{R}^{*}\right\}$

where

$\bar{A}=\sqrt{\frac{s q A \frac{(\vec{n} \cdot \vec{r})}{r}}{r^{2}}} \exp \{\vec{k} \cdot \vec{r}+\varphi\}$

is the wave, that is radiated by the elemental area (notations here are the same as in Eq. (11)). Wave

$\bar{R}^{*}=\operatorname{Re} x p\left\{j \eta \cdot x_{F H}\right\}-H D R W$

$\left(\bar{R}^{*}=\operatorname{Re} x p\left\{j \eta \cdot y_{i}\right\}-V D R W\right)$

is conjugated to the reference wave (HDRW means the horizontal incidence of the reference wave, VDRW - the vertical one). Assuming $R=1 / 2$, substituting (17), (18) into (16), and taking into consideration mutual overlapping elements of the object, one can deduce (11) and (15), respectively.

\subsection{Definition of optimal quantization parameters.}

During the synthesis $\mathrm{CGH}$ need discretization in space and quantization of amplitude for every count with using limited number of levels. In quantization process the count value is compared with the threshold levels $d_{i}$ standing over the quantization step $q$. If amplitude of counts get between two neighbour threshold levels $\left(d_{i} \leq T[r, s]<d_{i+1}\right)$, it get value of the fixed quantization level $r_{i}$ corresponding for the interval. It is known that the center of interval between two neighbour threshold levels is the optimal position of quantization level for quantization function

$r_{i}=\frac{\left(d_{i}+d_{i+1}\right)-1}{2}$ 


\section{V.I. Girnyk et al.: Multilevel computer-generated holograms ...}

in this case dispersion of quantization noise can be defined as

$$
\sigma_{0}^{2}=\frac{q^{2}}{12}
$$

This correlation corresponds to the so-called linear model, because it is obtained under presumption that the quantization noise source is additive and independent. Unfortunately, photoresist (phase-registering medium) that was used for $\mathrm{CGH}$ registration has essentially nonlinear characteristics, and it cannot be described by linear model for substantial phase shifts over $0.6 \mathrm{rad}$. Abundance of factors which influence on diffraction efficiency and SNR make it expedient to estimate these parameters applying numerical methods during modeling process and experimental methods during recording process. Fig. 3 presents the results of investigations of quantization levels number influence on reconstructed image quality. We can see that it is expedient to select $16 \leq q \ln \leq 128$ if it allows the dynamic range of EBL-photoresist system. It provides high quality of CGH under the low cost.

\subsection{Modified approach to object wave amplitude calculation}

In some cases, there is a necessity to record a hologram of an object or its part with high resolution. As an example, we can indicate the need to include microimages into 3D synthesized holographic protective elements, these microimages being not observed with the naked eye but could be simply controlled under sufficient optical magnification. To use the approach based on Eq. (15) for synthesizing such holograms, it is necessary to reduce the object space discretization step $h$ by 10 times or more. It results in respective increase of the number of point sources that represent the object surface. Accordingly, time expenses for calculations increase by $\left(h_{0} / h_{\mathrm{m}}\right)$ to $\left(h_{0} / h_{\mathrm{m}}\right)^{2}$ times as dependent on microobject complexity (here $h_{0} / h_{\mathrm{m}}$ is the ratio of discretization step for the main part and microobject, respectively). To overcome this problem and speed digital synthesis, we suggest using the next approach for calculation of microobject surface element contribution.

As known, Eq. (15) arises as a result of digital calculation of the Fresnel integral

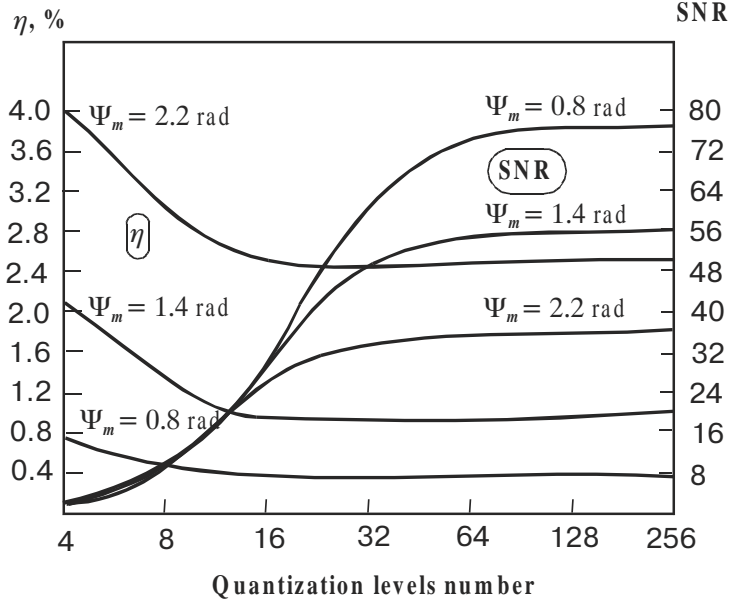

Fig. 3. Influence of quantization levels number on quality of reconstructed image.

$O(x)=\int \frac{A(\tilde{x}, \tilde{z})}{j \lambda r} \exp \{j k r+j \varphi\} d \tilde{x}$

by the trapezium method, and further extracting the informational part of interference of the obtained object wave amplitude with reference one. $(\tilde{x}, \tilde{z})$ is a point of the object space in the parallax plane, $r$ is a distance from this point to the point where the object wave amplitude $O(x)$ is calculated, $A(\tilde{x}, \tilde{z})$ is a wave amplitude on the object surface.

To calculate the object wave amplitude, we divide the object surface section by finite segments and integrate Eq. (21) analytically within each of these segments. To make the approximate estimation of the integral, we can use the Taylor expansion of the integral in the vicinity of the middle point $M$ of the each segment $[A, B]$ in the object section using smallness parameters

$\alpha=\frac{2 L}{r} \cos \theta, \beta=\frac{L^{2}}{r^{2}}$

For simplicity, we use an approximation of the section segment by a straight line, however, analogous expressions can be also deduced for any analytical curves that have a curvature radius sufficiently large compared to the segment length $L \cdot r$ is a length of a vector drawn from the point $x$ of the elemental hologram into the segment middle point $M$, and $\theta$ is an angle between vectors $\vec{r}$ and $\overrightarrow{A B}$

$$
\begin{aligned}
& O(x)=O_{0} \int_{-1 / 2}^{1 / 2}\left(1-\frac{1}{2} \alpha s+a_{2} s^{2}+a_{3} s^{3}+a_{4} s^{4}+\ldots\right) \exp \left\{j k r\left(\frac{1}{2} \alpha_{s}+b_{2} s^{2}+b_{3} s^{3}+b_{4} s^{4}+\ldots\right)\right\} d s \approx \\
& \approx O_{0} \int_{-1 / 2}^{1 / 2}\left(1-\frac{1}{2} \alpha s+a_{2} s^{2}+a_{3} s^{3}+a_{4} s^{4}\right)\left(1+j k r b_{2} s^{2}+j k r b_{3} s^{3}+\ldots\right) \exp \left\{j \frac{1}{2} k r \alpha s\right\} d s
\end{aligned}
$$




\section{V.I. Girnyk et al.: Multilevel computer-generated holograms ...}

where $O_{0}=\frac{A L}{j \lambda r} \exp \{j k r+i \varphi\}, k=\frac{2 \pi}{\lambda}$ is a wavenumber, $\lambda$ is a wavelength of radiation, $s$ is a curve parameter taking values $-1 / 2$ and $+1 / 2$ at the segment ends and zero in the middle; $a_{2}=\frac{3}{8} \alpha^{2}-\frac{1}{2} \beta, a_{3}=-\frac{5}{16} \alpha^{3}+\frac{3}{4} \alpha \beta$, $a_{4}=\frac{35}{128} \alpha^{4}-\frac{15}{16} \alpha^{2} \beta+\frac{3}{8} \beta^{2}, b_{2}=-\frac{1}{8} \alpha^{2}+\frac{1}{2} \beta, b_{3}=$ $=\frac{1}{16} \alpha^{3}-\frac{1}{4} \alpha \beta, b_{4}=-\frac{5}{128} \alpha^{4}+\frac{3}{16} \alpha^{2} \beta-\frac{1}{8} \beta^{2}$. The parameter $\gamma=k r \alpha=\frac{4 \pi L}{\lambda} \cos \theta$ is large $(>>1)$ and the latter exponent in the integral (23) is fast oscillating. The existence conditions of the representation (23) are as follows:

$$
\begin{aligned}
& \frac{L}{r}<<1, \\
& \frac{1}{4} k r b_{2}=\frac{\pi L^{2}}{4 \lambda r} \sin ^{2} \theta<<1, \\
& \frac{1}{8} k r b_{3} \approx \frac{\pi L^{3}}{8 \lambda r^{2}}|\cos \theta| \sin ^{2} \theta<<1
\end{aligned}
$$

The first condition is easily achievable, and the second and third ones give some limitations for the permissible segment length of integration. The term generating the condition (24) is taken into account during the following integration and included into a correction multiplier, therefore, its value does not contribute any supplementary error into the final result. It is sufficient to choose it to be approximately 0.4 for the expansion into series to take place. At given accuracy $1 \%$ and the wavelength $0.4 \mathrm{~mm}$, using the conditions (25) and (26) we obtain the next limitations: $L_{\max }<0.6 \mu \mathrm{m}$ at $r=10 \mathrm{~mm}$, $L_{\max }<3 \mu \mathrm{m}$ at $r=0.1 \mathrm{~mm}, L_{\max }<14 \mu \mathrm{m}$ at $r=1 \mathrm{~mm}$, $L_{\max }<45 \mu$ m at $r=10 \mathrm{~mm}$.

Then, multiplying expressions in parentheses in the latter integral in (23), and integrating it term-by-term, we obtain the following equation (keeping only the leading terms of the expansion):

$$
O(x)=\left[\frac{A L}{j \lambda r} \exp \{j k r+i \varphi\}\right]\left(1+j \frac{\pi L^{2}}{4 \lambda r} \sin ^{2} \theta\right)\left(\frac{\sin \gamma}{\gamma}\right)
$$

Here, the term in brackets coincides with the amplitude of the object wave from a point source localized in the middle of segment $[A, B]$. The second multiplier gives a correction to the amplitude, which does not exceed $40 \%$. And the latter multiplier is an interference term taking into account coherent summation of partial waves from the points of the segment $[A, B]$; in the limit, $\gamma \rightarrow 0$, which means the case of a point source, this multiplier transforms into unity.

Using the formula (27) enables to analytically sum contributions from the whole group of closely located point sources, distances between which are less than the value $L_{\max }$ deduced above. This allows us to shorten the calculation time by orders when synthesizing 3D hologram with high resolution.

\section{Basis and peculiarities of algorithm}

\subsection{Object representation}

With the aim of simplifying program means, we used model representations of objects in the form of a set of plane images having arbitrary orientations in the object space. However, this model representations enables to adequately investigate workability of the above approach in general, and even to emphasize realization peculiarities of characteristic variants for locating image elements within the object space. Investigations for optimization of all managing parameters can be completely carried out in the case of characteristic test elements of the object space within this model representation, and their results can be used in the following study with improved representations of the object, namely, not quasi-3D, but by the real bulk one. As to such improvement, to some extent, it can be made in the framework of using plane images, stretching them on simple surfaces that differ from the plane ones: cylindrical, spherical, conical, parabolical etc., but the valid bulk representation all the same is more desirable.

\subsection{Basic parts of the algorithm}

Algorithm is mainly composed from two parts: synthesis and recording. Synthesis stage implies calculation of the interferogram data, and the recording stage - its suitable transformation and registration. Fig. 4 shows the extended scheme of the main production algorithm. It is necessary to mention the influence of quantization parameters on obtained results. So, analysis of statistic characteristics of ID is sufficiently necessary.

\subsection{Looking for shielded points}

Separate parts of the object can be overlapped and shielded by one another. Shielded elements are not seen from the considered point of the elementary hologram (PEH) and do not contribute into an amplitude of the object wave. Various parts of the object are shielded for different PEHs. To find shielded elements of object, represented as a set of $N$ point sources, using the method of direct ray tracing needs $\sim N^{2}$ operations for each PEH. It increases the time of a hologram synthesis up to unacceptable values (approximately 1 year). To overcome this problem, we have developed the algorithm for fast searching these shielded object parts. It requires about $20 \mathrm{~N}$ operations with a floating point. To this aim, the object section is approximated by a broken line, each segment of which comprises one or several radiating sources. Using the known method of chain cells all segments are ordered along the z-coordinate and are stored in a $z$-buffer. 


\section{V.I. Girnyk et al.: Multilevel computer-generated holograms ...}

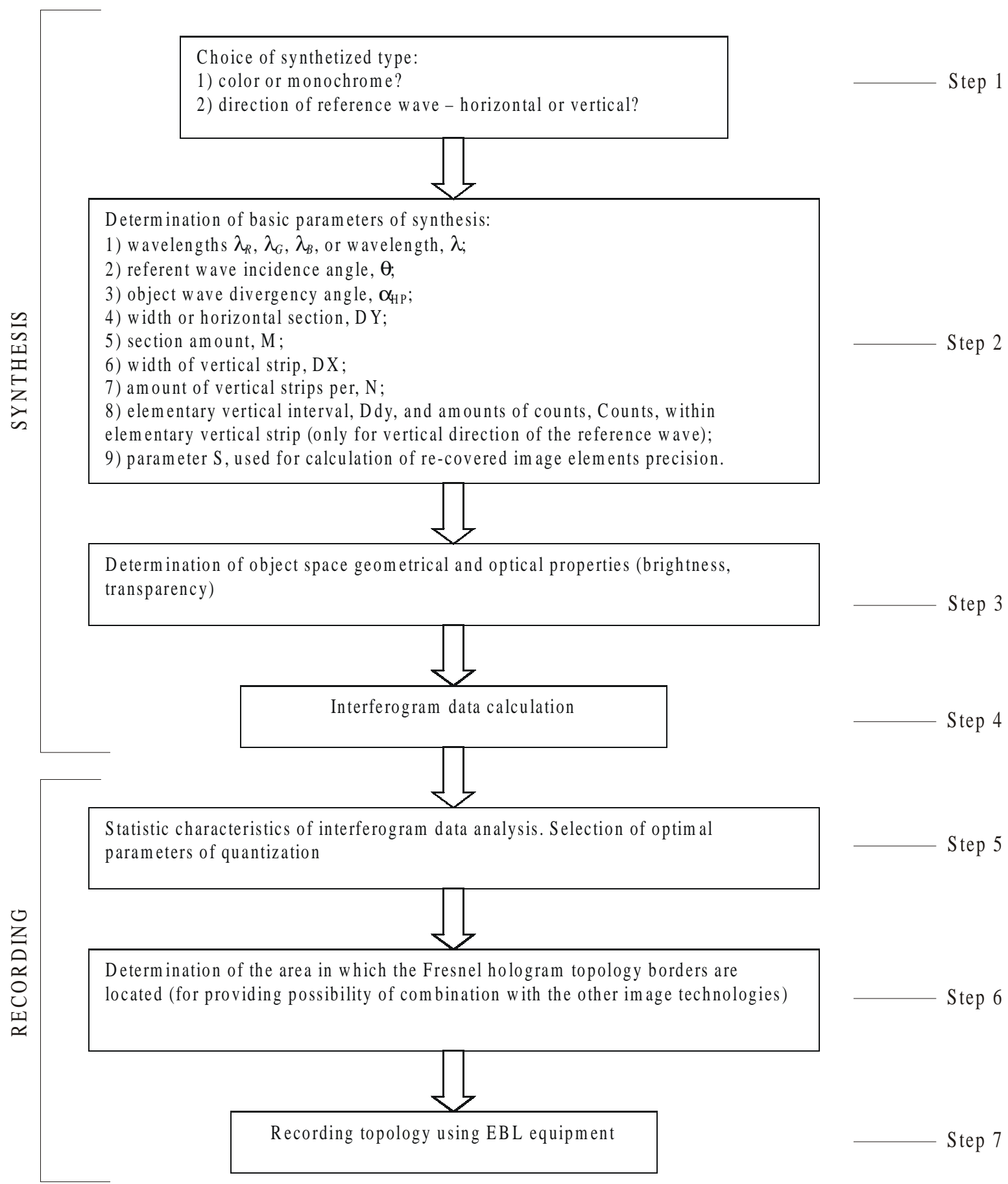

Fig. 4. Flowchart of macro-algorithm.

Each segment forms a shadow sector limited by rays passing through its extreme points $(A, B)$ and the point $x$ of elementary hologram. Object elements falling into the shadow sector are invisible from the point $x$.

The algorithm operates as follows. Object elements (i.e. section segment) taken from the $z$-buffer are sorted out in the order of increasing distances from the $x$-point. There are calculated angle equivalents for rays limiting the shadow sector of that segment. The angle equivalents are integers, they are calculated using a special algorithm without calling trigonometrical functions. The value of a visibility coefficient for a given section element is estimated throw the degree of overlapping the sector, confined by its limiting angles, with a total shadow zone. After this, the total shadow zone is enlarged by this sector. At a computer with the clock frequency $1000 \mathrm{MHz}$ the algorithm work time can be reduced down to $25 . . .35$ ms $\left(\right.$ for $\left.N=10^{5}\right)$. 


\section{V.I. Girnyk et al.: Multilevel computer-generated holograms ...}

\section{Experimental results}

Results of investigations of test hologram recording are presented in the next figures. Synthesized hologram of test object (logotype "OES" splitted at 3 planes) has been printed on electron resist and on $\mathrm{As}_{40} \mathrm{~S}_{40} \mathrm{Se}_{20}$ photoresist $[8,9,10]$ by EBL device. Structure of resist relief has been investigated by using electron beam microscope.

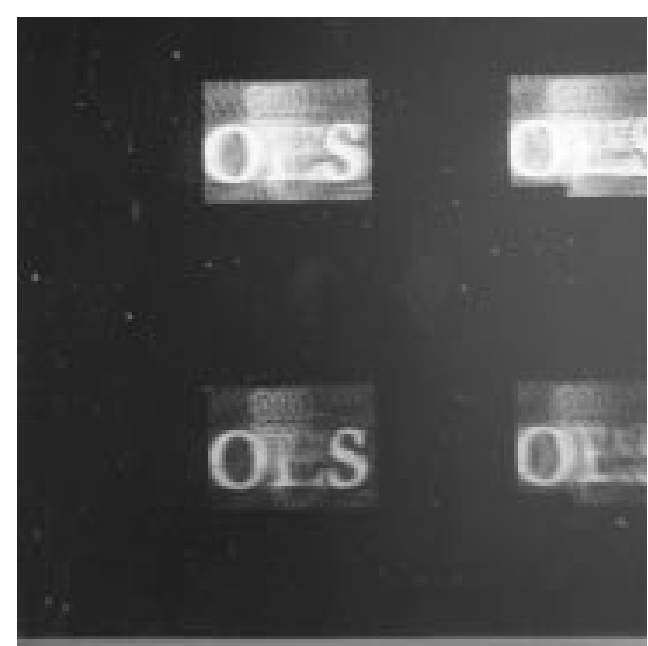

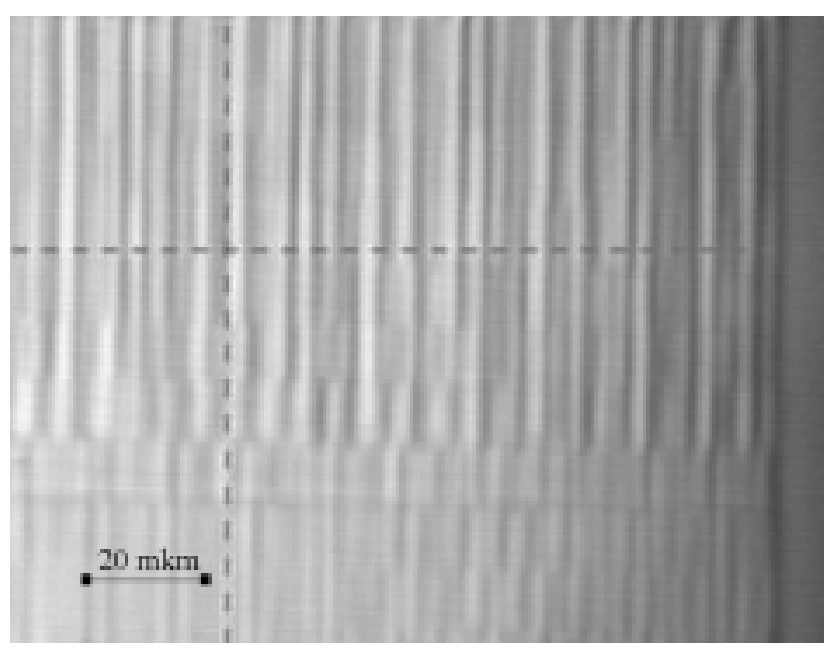

Fig. 5. Analysis of the hologram recorded using non-organic resist on different scale levels. a) Examples of holograms recorded with different exposures which corresponds to different phase shifts. b) Microstructure of the hologram recorded by EBL.
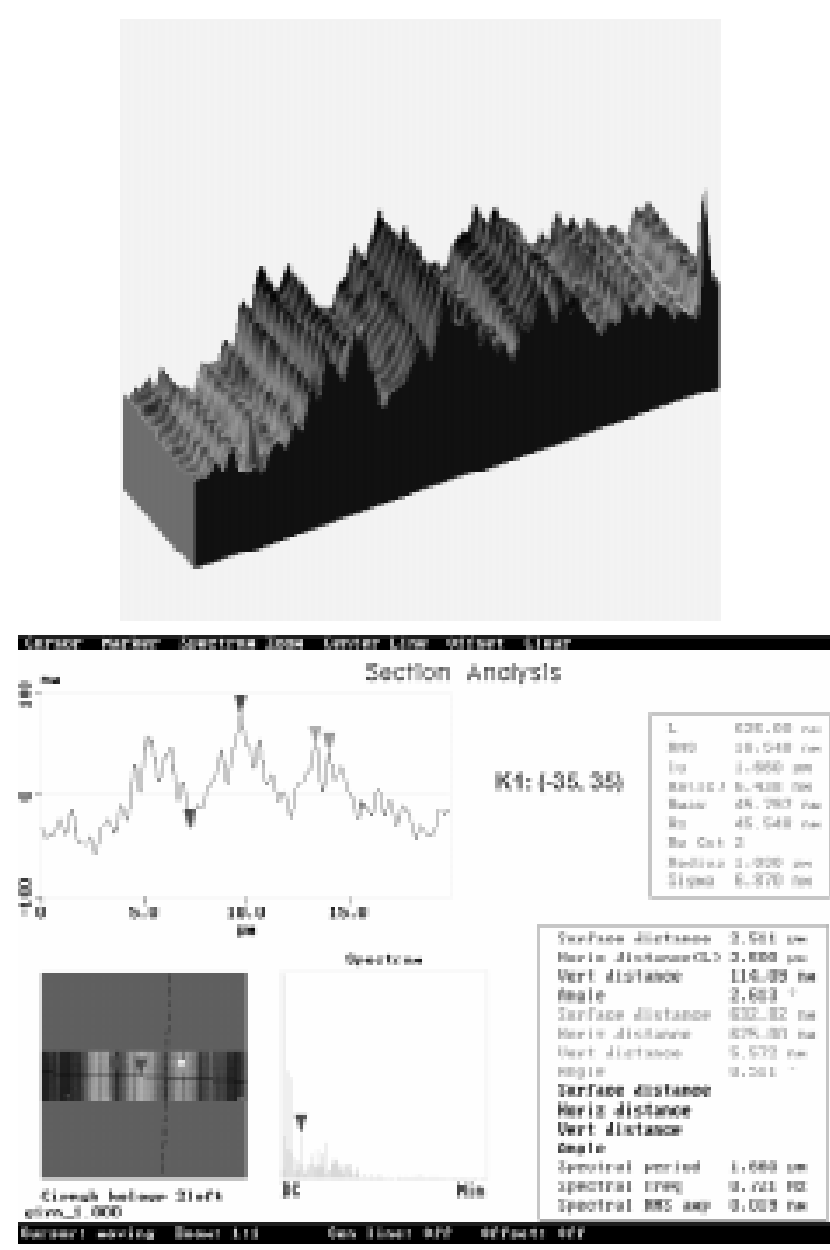

$\mathbf{a}$

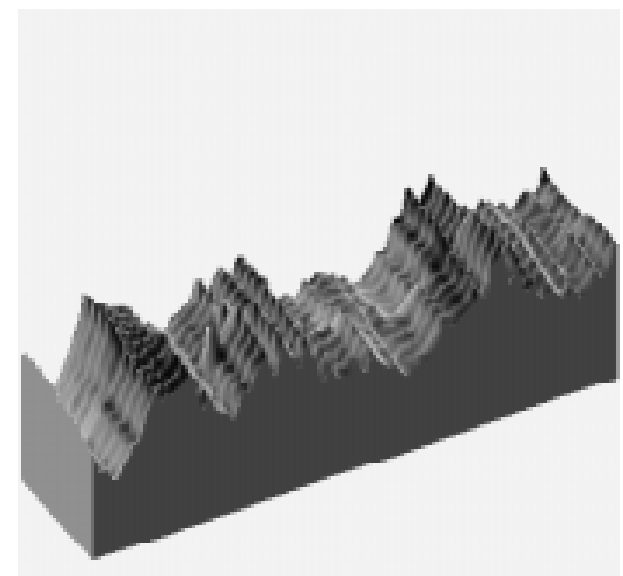

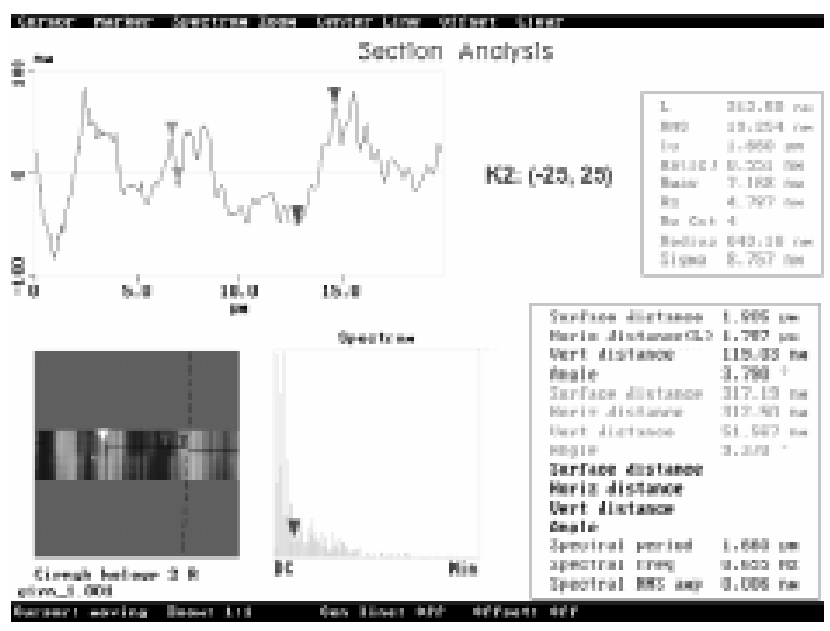

b

Fig. 6. Influence of quantization method. a) linear quantization. b) nonlinear quantization with cutting amplitudes deviation of which from the middle value exceeds some limit. Both relief are made using non-organic resist $\mathrm{As}_{40} \mathrm{~S}_{40} \mathrm{Se}_{20}$. 


\section{V.I. Girnyk et al.: Multilevel computer-generated holograms ...}
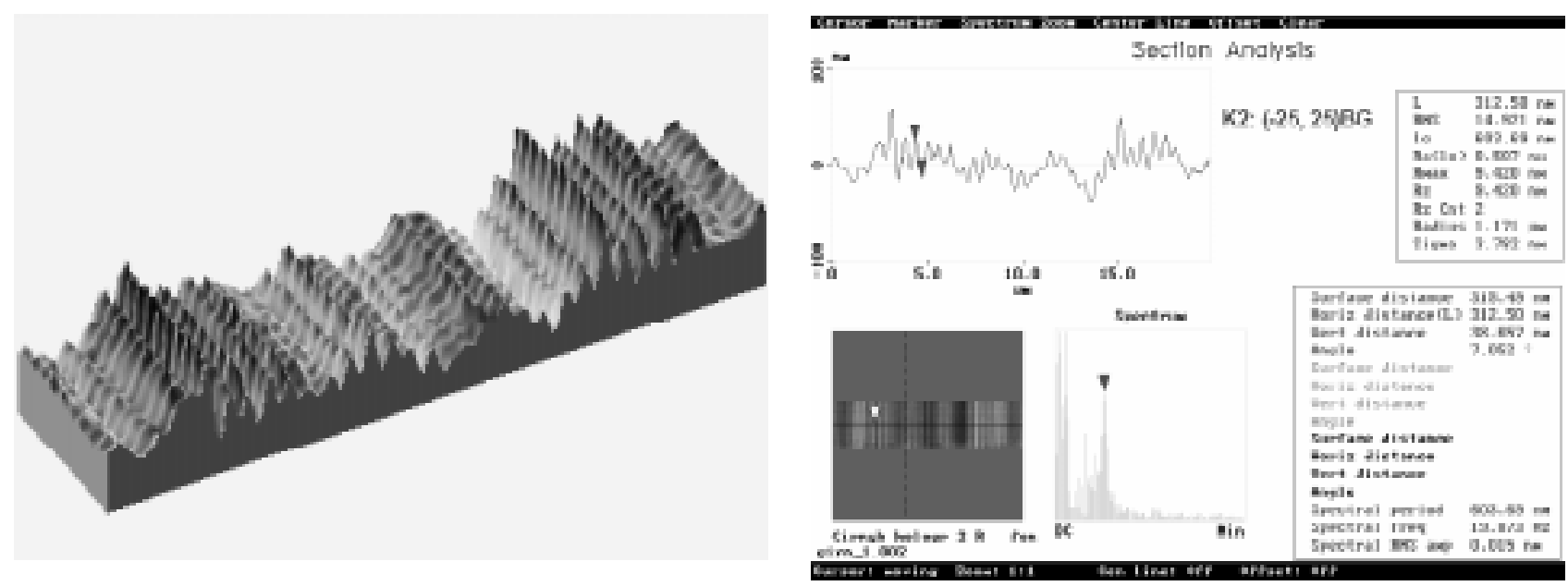

Fig. 7. The example of a non-optimal fragment of the CGH recording using non-organic resist $\mathrm{As}_{40} \mathrm{~S}_{40} \mathrm{Se}_{20}$.

\section{Conclusions}

We had carried out investigations on the advantages of the original approach which imply representation of an object surface as the aggregation of the elemental selfradiating areas in comparison with the traditional approach of representation of an object as the point light sources aggregation. Investigations was carried out on quality level and gave us positive results. Quantitative evaluation of the advantages is planned in the nearest future. Investigations on the optimization of quantization parameters was also carried out. It was ascertained that the number of quantization levels of 16 value is optimal for the AsGa registering medium in the application of the recording of CGH of $3 \mathrm{D}$-images. We have to mention that there still remains a need in the determination of the appropriate parameters of count discretization in CGH plane for characteristic parameters of recording scheme, and also in the optimization of object parameters for CGH of different types (monochrome and color) and of any reference wave direction.

\section{References}

1. Girnyk V.I., Tverdochleb I.V., Ivanovsy A.A., Combined Optical/Digital Security Devices, SPIE 3973, pp. 322-327, 2000.

2. T. Hamano, H. Yoshikawa, "Image-type CGH by means of e-beam printing, SPIE 3293, pp.2-14, 1998.

3. T. Hamano, H. Yoshikawa, Computer-generated holograms with pulse-width modulation for multi-level 3D images," SPIE 3637, pp. 244-251, 1999.

4. T. Hamano, M. Kitamura, Computer-generated holograms for reconstructing multi 3D images by space-division recording method, SPIE 3956, pp. 23-32, 2000.

5. Mark Lucente, Interactive computation of holograms using a look-up table, SPIE 1667, 1992.

6. D. Leseberg, Computer-generated three-dimensional image holograms, Appl. Opt. 31,2, pp. 223-229, 1992.

7. A. W. Lohmann, S. Sinzinger, Graphic codes for computer holography, Appl. Opt. 34,17, pp. 3172-3178, 1995.

8. Solution for negative etching chalcogenide glasses, Patent on Invention № 2008285, Russian Federation, Indutnyy I.Z., Kostyukevych S.O., Shepeliavyi P.E., priority from 20.06.91, Bulletin 4, 28.02.94.

9. The way of manufacturing holographic diffraction gratings, Patent on Invention № 2165637, Russian Federation, Venger E.F., Kostyukevych S.O., Shepeliavyi P.E., Goltsov Yu.G., priority from 17.11.1999, Bulletin 11, 20.04.2001.

10. The way of manufacturing holographic diffraction gratings, Decraration Patent on Invention № 36209 A, Ukraine, Venger E.F., Kostyukevych S.O., Shepeliavyi P.E., Goltsov Yu.G., priority from 17.11.1999, Bulletin 3, 16.04.2001. 